\title{
Prognostic factors of unresectable hepatocellular carcinoma treated with yttrium-90 radioembolization: results from a large cohort over 13 years at a single center
}

\author{
Rucha M. Shah ${ }^{\wedge}$, Sarah Sheikh², Jimmy Shah ${ }^{3}$, Elaina Vivian², Alejandro Mejia ${ }^{2}$, Islam Shahin ${ }^{4}$, \\ Parvez S. Mantry ${ }^{2} \wedge$
}

${ }^{1}$ Gastroenterology and Hepatology, Methodist Dallas Medical Center, Dallas, TX, USA; ${ }^{2}$ The Liver Institute, Methodist Dallas Medical Center, Dallas, TX, USA; ${ }^{3}$ Methodist Digestive Institute, Methodist Dallas Medical Center, Dallas, TX, USA; ${ }^{4}$ Interventional Radiology, Methodist Dallas Medical Center, Dallas, TX, USA

Contributions: (I) Conception and design: RM Shah, E Vivian, A Mejia, I Shahin, PS Mantry; (II) Administrative support: PS Mantry; (III) Provision of study materials or patients: PS Mantry, RM Shah, J Shah, I Shahin; (IV) Collection and assembly of data: RM Shah, S Sheikh, J Shah, A Mejia, I Shahin, PS Mantry; (V) Data analysis and interpretation: RM Shah, PS Mantry; (VI) Manuscript writing: All authors; (VII) Final approval of manuscript: All authors.

Correspondence to: Parvez S. Mantry, MD, AGAF, FAASLD. The Liver Institute, Methodist Dallas Medical Center, 1411 Beckley Ave \#268, Dallas, TX 75203, USA. Email: parvezmantry@mhd.com.

Background: A previous study of patients with unresectable hepatocellular carcinoma (HCC) was extended to further examine factors associated with overall survival (OS) after selective internal radiation therapy with yttrium-90 resin microspheres (Y90 SIRT).

Methods: Data from patients of any age diagnosed with unresectable HCC and treated with Y90 SIRT at our institution from 2004 through 2017 were retrospectively analyzed. Among other criteria, patients had to have Eastern Cooperative Oncology Group performance status 0 to 2, not have received Y90 SIRT previously, and not have extrahepatic disease. Primary outcome was OS; secondary outcomes included tumor response and adverse events (AEs). Kaplan-Meier survival analyses and multivariable Cox proportional hazards models were used to evaluate prognostic factors for OS.

Results: Of the 226 patients, 59\% were White, 77\% were male, and the mean age at first SIRT procedure was $65.1 \pm 9.4$ years. More than half had received previous treatment for HCC. The most common etiology was hepatitis C ( $\mathrm{n}=138 / 224$ available, $62 \%)$, followed by alcohol use $(\mathrm{n}=45,20 \%)$, and nonalcoholic steatohepatitis $(\mathrm{n}=27,12 \%)$. The mean model for end-stage liver disease score at baseline was 8.8 \pm 2.2 . Patients were followedup for a median of 12.2 months (95\% CI, 0.0-62.6). Median OS was 16.6 months (95\% CI, 13.1 to not reached). Bilobar disease, higher albumin-bilirubin score at baseline, prior treatment with sorafenib, alcohol use etiology, and higher administered dose were associated with shorter survival, whereas subsequent liver transplant [in 26 patients (11.5\%)] was associated with longer survival. Of the 186 patients with AEs data, 75 $(40.3 \%)$ patients reported an event and, of these, 13 (17.3\%) patients had grade 4 bilirubin values.

Conclusions: In a large, diverse population treated at a single center over 13 years, Y90 SIRT produced a median OS of 16.6 months in patients with unresectable HCC and enabled subsequent transplantation in a subset of patients. Factors affecting the length of survival should be considered when making treatment decisions for unresectable HCC.

Keywords: Radioembolization; selective internal radiation therapy (SIRT); liver-directed therapy; locoregional treatment; liver cancer

^ ORCID: Rucha M. Shah, 0000-0001-8538-8021; Parvez S. Mantry, 0000-0003-4829-6931. 
Submitted Oct 09, 2020. Accepted for publication Jun 08, 2021.

doi: 10.21037/jgo-20-435

View this article at: https://dx.doi.org/10.21037/jgo-20-435

\section{Introduction}

Hepatocellular carcinoma (HCC) is the most common form of liver cancer $(1,2)$. The incidence of HCC has historically been the highest in Asia and Africa and the lowest in Europe and the Americas $(3,4)$, but the incidence in the United States rose about $3 \%$ per year from 2006 to 2015 and has more than tripled since 1980, although this increase seems to be leveling off (1). Prognosis of liver cancer is poor, with only $20 \%$ of patients in the United States surviving past 5 years (5). Curative treatments are not an option for the majority of patients (up to $70 \%$ ) due to their stage at diagnosis (6). Patients diagnosed with distant disease, in particular, have a very low 5 -year survival rate of $3 \%$ (5). Other factors besides stage have been shown to significantly affect the prognosis of patients with HCC, including tumor size, presence of portal vein thrombosis, Child-Pugh class, and albumin-bilirubin (ALBI) score (7-9). Understanding the role of prognostic factors is useful to stratify patients to receive more appropriate treatments (10).

Underlying liver dysfunction almost always precedes HCC $(11,12)$ and also affects its prognosis $(13)$. This presents a major challenge in the care of patients with HCC because the impact of treatment on liver function must be taken into account. The stage of HCC at diagnosis affects the choice of initial treatment. Curative treatments (e.g., resection, transplantation, ablation for small lesions) are only effective at early stages (14) or after downstaging with other therapies (15). Approved systemic therapies for firstline treatment of HCC, such as sorafenib and lenvatinib $(14,16,17)$, can only be used in patients with relatively preserved liver function (Child-Pugh A or B) (14). Immune checkpoint inhibitors nivolumab and pembrolizumab were recently approved for patients previously treated with sorafenib $(18,19)$, and additional immunotherapies are being explored, but these are also primarily used in patients with good liver function (i.e., Child-Pugh A) $(20,21)$, and after failure of first-line chemotherapy.

Locoregional therapies (ablation, arterially directed therapies, radiation) are the preferred, and often only, first-line treatments for unresectable HCC (14). Selective internal radiation therapy with yttrium-90 microspheres
(Y90 SIRT) - made of glass or resin-is a locoregional therapy that delivers radioactive microspheres to the tumor via the hepatic artery, sparing much of the healthy liver tissue (22).

Previous large ( $>100$ patients) prospective and retrospective studies of Y90 SIRT in patients with unresectable HCC have found an overall survival (OS) of 8.0 to 14.4 months after Y90 SIRT (23-27). Although factors related to the patient, disease, and/or treatment have the potential to affect outcomes after Y90 SIRT, more data are needed to determine which patients will benefit the most from this therapy (13). Not all of the abovementioned studies that treated more than 100 patients with resin-based SIRT evaluated prognostic factors (26), and 1 study did so as a post hoc analysis of a subgroup of patients (28). Prognostic factors have also been identified in smaller studies (34-77 patients) (29-33), with various limitations in addition to their small size such as evaluation of few factors (30), evaluation only comparing SIRT with another treatment (29), evaluation combining HCC with other malignancies (30), and lack of multivariate analysis (33). Relatively large studies (120-149 patients) using Y90 SIRT glass microspheres for HCC have also identified prognostic factors and created prognostic scores $(34,35)$; however, extrapolation of these findings to patients treated with Y90 SIRT resin microspheres may be challenging. Overall, there is high variability between studies, with many basing their conclusions on a small group of patients.

We previously reported an OS of 13.1 months (95\% CI, 10.3-18.4) in 111 patients with HCC treated with Y90 SIRT at our center from April 2004 through March 2013 (27), and found that bilobar disease, presence of PVT or ascites at baseline, and prior treatment with sorafenib were among the factors that predicted shorter OS. Here, we have extended this study to include additional follow-up on the original group of patients, as well as patients treated from April 2013 through March 2017. This more than doubled the number of participants studied initially and increased the precision with which we could estimate OS and detect factors associated with survival after Y90 SIRT. The description of the study is presented in accordance with the STROBE reporting checklist (available at https:// dx.doi.org/10.21037/jgo-20-435). 


\section{Methods}

\section{Patient selection}

The study population for this retrospective cohort included all patients with unresectable HCC who were treated with Y90 SIRT at Methodist Dallas Medical Center from April 2004 through March 2017. Patients were followed-up for a median of 12.2 months (95\% CI, 0.0-62.6). One patient was not followed-up post-SIRT treatment and was assigned follow-up of 1 day. HCC was considered unresectable if the tumors were multifocal or bilobar or if PVT, portal hypertension, or decompensated liver disease (Child-Pugh $\mathrm{B}$ or C) were present.

To be eligible for the Y90 SIRT procedure, patients needed to have Eastern Cooperative Oncology Group performance status 0 to 2 ; platelet count $>6.0 \times 10^{4} / \mu \mathrm{L}$; creatinine level $<2 \mathrm{mg} / \mathrm{dL}$; bilirubin level $<2 \mathrm{mg} / \mathrm{dL}$; and international normalized ratio (INR) $<1.2$. Patients with a contraindication to hepatic artery catheterization such as vascular abnormalities, bleeding diathesis, allergy to contrast dye, concurrent malignancy, refractory ascites, or evidence of any uncorrectable flow to the gastrointestinal tract were not eligible to undergo Y90 SIRT. An estimation based on angiography or technetium-99m macroaggregated albumin scan that $>30$ Gy of radiation would be delivered to the lung (shunt fraction of $20 \%$ or greater) also precluded treatment with Y90 SIRT.

Patients treated with Y90 SIRT were excluded from this study if data were only available for $<20 \%$ of the parameters, if the patient had undergone a prior Y90 SIRT procedure at a different facility, or if extrahepatic disease was present.

\section{Y90 SIRT protocol}

Transplant hepatologists led a multidisciplinary treatment team that also included oncologists, surgeons, hepatologists, and interventional and cross-sectional radiologists. The medical history of each patient was reviewed, and a physical examination and laboratory testing were performed.

To confirm that Y90 SIRT was appropriate, multiphasic computed tomography or hepatic magnetic resonance imaging was performed to determine portal vein patency, tumor and nontumor liver volume, and the presence or absence of extrahepatic tumors. The images also assisted in targeting the radioembolization and determining the appropriate activity to administer. Y90 resin microspheres (SIR-Spheres, Sirtex Medical Inc, Sydney, Australia) were administered in accordance with a standard protocol (36). Radiographic data were read by one of the authors (IS) together with a group of radiologists at the medical center where the procedures were carried out.

\section{Data collection}

Patient data were collected by retrospective chart review. Data from patients treated from April 2004 through March 2013 were analyzed and published previously (27). Data from patients followed or treated from March 2013 through March 2017 were abstracted separately and combined with the previous data set.

Baseline data were collected at the time of the first SIRT and included demographics, HCC etiology, disease state [tumor burden; model for end-stage liver disease (MELD) score; Child-Pugh score; presence of PVT or ascites], and laboratory values for albumin, bilirubin, creatinine, and INR. ALBI score and grade (37) were calculated. The number of Y90 SIRT treatments, as well as the dates and administered dose of each treatment, were recorded.

\section{Outcomes}

The primary outcome was OS, defined as the time (months) from the first Y90 SIRT procedure until death or last follow-up; patients alive at last follow-up were censored at the last known date they were alive.

Secondary outcomes were as follows: (I) the tumor response based on imaging data collected at 3 months and 6 months after the first SIRT procedure, when available. Tumor response (complete response, partial response, stable disease, or progressive disease) was calculated in accordance with the Response Evaluation Criteria in Solid Tumors (RECIST) (38) or modified RECIST (39); (II) relationship of administered dose to baseline MELD score and ALBI score; (III) relationship of prior treatment to tumor burden and MELD score at baseline; (IV) adverse events (AEs).

Descriptive information on AEs at 1 week and 3 months after the first Y90 SIRT was extracted from the charts and is reported as the number and percentage of patients who had the $\mathrm{AE}$ at either time point or at both. In addition, we examined the change in laboratory values from baseline to 1,3 , and 6 months after the first SIRT procedure and noted the number of laboratory values that were of Common Terminology Criteria for Adverse Events, version 3.0, grade 3 or above. Grades were not noted for other AEs because they were rarely included in the charts. 


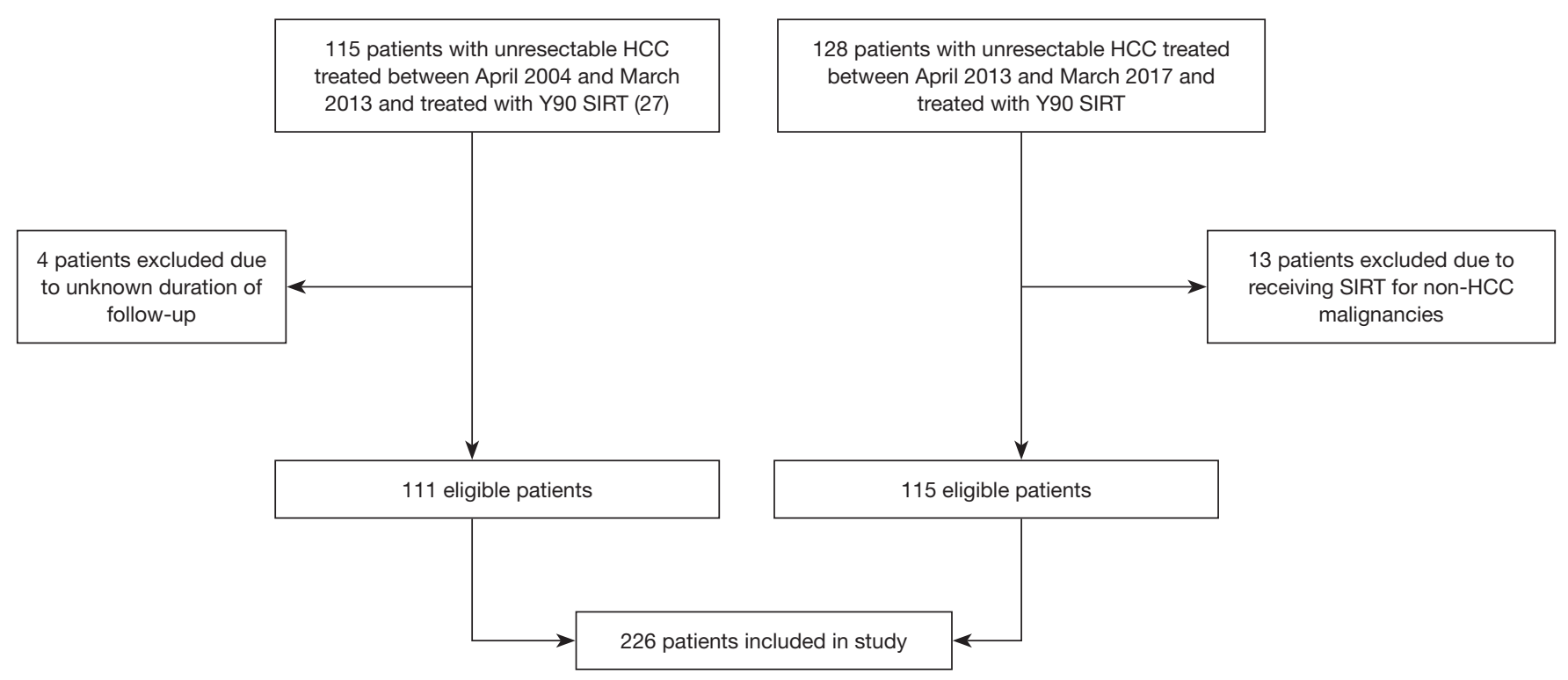

Figure 1 Flowchart of patient inclusion in study. HCC, hepatocellular carcinoma; Y90 SIRT, yttrium-90 selective internal radiation therapy.

\section{Statistical analysis}

Continuous variables were summarized as the mean \pm SD. Categorical data were summarized as frequencies and percentages. OS is reported as the median and 95\% CI. Between-group OS was analyzed with the Kaplan-Meier method using the log-rank test to determine significance, with $\mathrm{P}<0.05$ set as the threshold for significance.

OS was further analyzed with Cox proportional hazards models. Prognostic variables were analyzed in a univariate model, and the results were summarized with the hazard ratio, the $95 \% \mathrm{CI}$, of the hazard ratio, and a $\mathrm{P}$ value. Variables with $\mathrm{P}<0.1$ were then entered into a multivariable Cox model. Variable selection was achieved by backward elimination with a significance level of $\alpha=0.05$. Transplantation was included in the multivariable model as a time-dependent variable. As a sensitivity analysis, a multiple imputation procedure was implemented involving 100 complete datasets of 226 participants to address the influence of missing data.

For the secondary outcomes, the relationship of dose to baseline MELD score and dose to ALBI score was analyzed with the Pearson correlation coefficient. Tumor burden at baseline by prior treatment received versus treatment not received was analyzed with a 2 -sample $t$-test. MELD score by prior treatment received versus treatment not received was analyzed with a 2 -sample $t$-test, and changes in laboratory variables from baseline were tested with a paired $t$-test.

SAS (SAS Institute Inc.) version 9.4 was the main statistical package used for statistical analysis and reporting.

\section{Ethical statement}

The study was conducted in accordance with the Declaration of Helsinki (as revised in 2013). The study was approved by the Institutional Review Board of Methodist Dallas Medical Center (No. 063.HEP.2017.D), and individual consent for this retrospective analysis was waived.

\section{Results}

\section{Patients}

Data from a total of 226 patients were included in the study (Figure 1). The mean age at first Y90 SIRT was 65.1 years (range, 40.3-85.2); about one-third $(n=70)$ were aged $\geq 70$ years (Table 1 ). About three-quarters of the patients $(n=174,77 \%)$ were male, and just more than half $(\mathrm{n}=133,58.8 \%)$ were White. Patients were followed-up for 12.2 months (95\% CI, 0.0-62.6).

Hepatitis $\mathrm{C}$ virus (HCV) was by far the most common etiology $(n=138,61.6 \%$ of those with available data), followed by alcohol use ( $n=45,20.1 \%)$, and nonalcoholic 
Table 1 Demographic, disease, and treatment characteristics

\begin{tabular}{|c|c|}
\hline Characteristic & Value \\
\hline \multicolumn{2}{|l|}{ Sex, $n(\%), N=226^{\dagger}$} \\
\hline Female & $52(23.0)$ \\
\hline Male & $174(77.0)$ \\
\hline \multicolumn{2}{|l|}{ Race, n (\%), N=226 } \\
\hline American Indian & $1(0.4)$ \\
\hline Asian & 39 (17.3) \\
\hline Black & 27 (11.9) \\
\hline Hispanic & $20(8.8)$ \\
\hline White & $133(58.8)$ \\
\hline Other & $6(2.7)$ \\
\hline \multicolumn{2}{|c|}{ Age at first SIRT, years, $N=226$} \\
\hline Mean (SD) & $65.1(9.42)$ \\
\hline Range & $40.3-85.2$ \\
\hline$<70, \mathrm{n}(\%)$ & $156(69.0)$ \\
\hline$\geq 70, \mathrm{n}(\%)$ & $70(31.0)$ \\
\hline \multicolumn{2}{|c|}{ HCC etiology, n (\%), N=224 } \\
\hline Hepatitis C & $138(61.6)$ \\
\hline Alcohol use & $45(20.1)$ \\
\hline $\mathrm{NASH}$ & $27(12.1)$ \\
\hline Hepatitis B & $17(7.6)$ \\
\hline Cryptogenic & $15(6.7)$ \\
\hline Other ${ }^{\S}$ & $7(3.1)$ \\
\hline \multicolumn{2}{|l|}{ MELD score, N=218 } \\
\hline Mean (SD) & $8.8(2.2)$ \\
\hline$\leq 10, \mathrm{n}(\%)$ & $162(74.3)$ \\
\hline$>15, \mathrm{n}(\%)$ & $1(0.5)$ \\
\hline ALBI score, mean (SD) & $-2.3(0.57)$ \\
\hline \multicolumn{2}{|c|}{ Child-Pugh grade, $n(\%), N=150$} \\
\hline$A$ & $110(73.3)$ \\
\hline B & $37(24.7)$ \\
\hline C & $3(2.0)$ \\
\hline \multicolumn{2}{|c|}{ Target lesion size, $\mathrm{cm}, \mathrm{N}=114$} \\
\hline Mean (SD) & $6.8(4.4)$ \\
\hline Median (IQR) & $5.8(5.4)$ \\
\hline Range & $0.6-30.1$ \\
\hline
\end{tabular}

Table 1 (continued)

\begin{tabular}{lc}
\hline Characteristic & Value \\
\hline Bilobar disease, $\mathrm{n}(\%), \mathrm{N}=221$ & $105(47.5)$ \\
Portal vein thrombosis, $\mathrm{n}(\%)$, & $44(21.6)$ \\
$\mathrm{N}=204$ & \\
Ascites, $\mathrm{n}(\%), \mathrm{N}=211$ & $71(33.7)$ \\
Laboratory values, mean (SD) & \\
Bilirubin, $\mathrm{mg} / \mathrm{dL}, \mathrm{N}=226$ & $1.1(0.5)$ \\
Albumin, $\mathrm{g} / \mathrm{dL}, \mathrm{N}=224$ & $3.6(0.6)$ \\
Creatinine, $\mathrm{mg} / \mathrm{dL}, \mathrm{N}=220$ & $0.9(0.3)$ \\
INR, $\mathrm{N}=219$ & $1.1(0.2)$
\end{tabular}

Previous treatments, $\mathrm{n}(\%)$, $\mathrm{N}=179^{\text {? }}$

Any treatment

131 (73.2)

Transarterial chemoembolization

$74(41.3)$

Sorafenib

$40(22.4)$

Ablation

$25(14.0)$

Resection

21 (11.7)

Radiation

Y-90 resin treatments, $\mathrm{n}(\%), \mathrm{N}=226$

1

157 (69.5)

2

55 (24.3)

3

$10(4.4)$

4

Mean (SD)

$1.4(0.7)$

Radiation dose of first SIRT, $\mathrm{mCi}$, $\mathrm{N}=222$

Mean (SD)

$1.18(0.49)$

${ }^{\dagger}$, number of patients with available data is indicated; percentages are relative to the number with available data. ${ }^{\ddagger}$, some patients had $>1$ etiology. ${ }^{\S}$, "other" includes autoimmune hepatitis, iron-overload cirrhosis, primary biliary cirrhosis, de novo HCC. ", some patients had $>1$ previous treatment. ALBI, albumin-bilirubin; HCC, hepatocellular carcinoma; INR, international normalized ratio; IQR, interquartile range; $\mathrm{NASH}$, nonalcoholic steatohepatitis; max, maximum; MELD, model for end-stage liver disease; min, minimum; SD, standard deviation; SIRT, selective internal radiation therapy; Y-90, yttrium-90.

Table 1 (continued) 


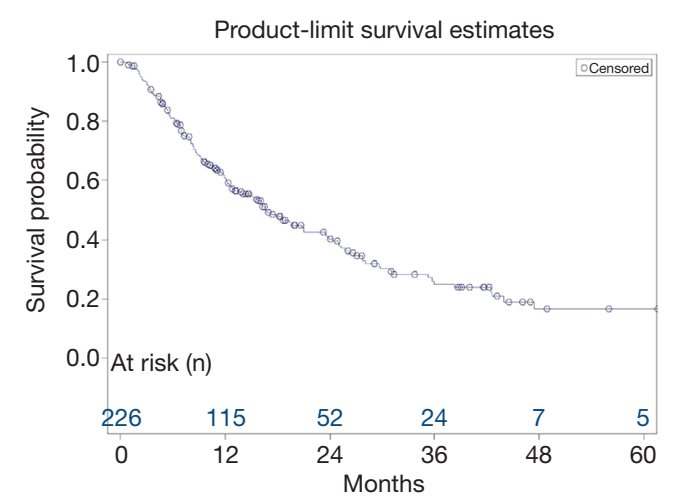

Figure 2 Kaplan-Meier survival curve. Survival of the total population $(\mathrm{N}=226)$ is shown.

steatohepatitis (NASH) $(\mathrm{n}=27,12.1 \%)$. Most patients had compensated or mildly decompensated liver disease, as indicated by laboratory values, MELD scores, ALBI scores, and Child-Pugh grades (Table 1).

Among the 179 patients with data on previous treatments for HCC, $131(73.2 \%)$ had received at least 1 treatment (Table 1); for 48 patients (26.8\%), Y90 SIRT was the first treatment. Transarterial chemoembolization (TACE) was the most common prior treatment $(\mathrm{n}=74,41.3 \%)$.

\section{Y90 SIRT}

The majority of the patients (157/226, 69.5\%) had a single Y90 SIRT procedure. Patients with bilobar disease had each lobe treated separately, which was counted as 2 procedures. Mean dose $( \pm$ SD) administered for the first Y90 SIRT procedure was $1.18 \pm 0.49 \mathrm{mCi}$.

\section{Primary outcome: $O S$}

At the time of the analysis, 138 patients had died. Median OS for all patients was 16.6 months (95\% CI, 13.1 to NR) (Table S1, Figure 2).

\section{Kaplan-Meier and log-rank analysis}

A Kaplan-Meier/log-rank analysis identified variables in several categories that were significantly associated with survival (Table S1). The disease characteristics of bilobar disease and the presence of PVT or ascites at baseline were all associated with shorter survival [bilobar: 10.8 months (95\%
CI, 8.5-15.2) vs. 25.0 months (95\% CI, 16.7-29.7), $\mathrm{P}<0.001$; PVT: 11.0 months (95\% CI, 8.0-13.9) vs. 19.3 months (95\% CI, 15.6-26.1), $\mathrm{P}=0.004$; ascites: 12.0 months (95\% CI, 8.417.9) vs. 20.8 months (95\% CI, 16.2-26.1), $\mathrm{P}=0.015$ ]. Alcohol use was the only etiology significantly associated with OS [11.8 months (95\% CI, 7.9-23.5) vs. 18.6 months (95\% CI, 15.6-24.4), $\mathrm{P}=0.02]$.

Among prior treatments, TACE was associated with longer survival [25.3 months (95\% CI, 13.9-29.8) vs. 13.5 months (95\% CI, 10.3-20.8), $\mathrm{P}=0.047$ ], whereas prior treatment with sorafenib was associated with shorter survival [10.3 months (95\% CI, 5.3-12.5) vs. 23.8 months (95\% CI, 16.2-27.8), $\mathrm{P}<0.001]$. However, any prior treatment versus Y90 SIRT as a first-line treatment was not significantly associated with length of OS.

Patients who underwent liver transplant after the SIRT procedure $(\mathrm{n}=26)$ had substantially longer survival (69.0 months, 95\% CI, 43.9-69.0) than patients who did not (13.9 months, 95\% CI, 11.8-16.7) $(\mathrm{P}<0.001)$.

\section{Cox proportional hazards models}

Variables with $\mathrm{P}<0.1$, the threshold for inclusion in the multivariable model, included measures of disease burden and liver function at baseline (bilobar disease, PVT, ascites, Child-Pugh score, laboratory markers of liver function), etiology of hepatitis B or alcohol use, prior treatment with sorafenib or TACE, administered dose, and subsequent transplant (Table 2).

In the multivariable model, bilobar disease, higher ALBI score at baseline, alcohol use etiology, prior treatment with sorafenib, and higher administered dose were all significantly associated with shorter OS, whereas subsequent liver transplant was significantly associated with longer survival (Figure 3). The sensitivity analysis by multiple imputation demonstrated the same direction of associations. Higher administered dose did not reach statistical significance in the sensitivity analysis (Table S2).

\section{Tumor response}

Of the 226 patients, $122(54.0 \%)$ and 105 (46.5\%) had imaging results available at 3 and 6 months, respectively. Of those with available data, at 3 months, 25 (20.5\%) had a complete response, 29 (23.8\%) had a partial response, 40 (32.8\%) had stable disease, and 28 (23.0\%) had progressive 
Table 2 Univariate Cox proportional hazards model

\begin{tabular}{|c|c|c|c|}
\hline Parameter & HR & $95 \% \mathrm{Cl}$ & $\mathrm{P}$ \\
\hline Sex & 0.80 & $0.54,1.17$ & 0.252 \\
\hline White & 1.11 & $0.79,1.56$ & 0.557 \\
\hline Age $\geq 65$ (years) & 1.07 & $0.77,1.50$ & 0.675 \\
\hline Age at diagnosis (continuous) & 1.01 & $0.99,1.03$ & 0.468 \\
\hline Age at 1st SIRT (continuous) & 1.00 & $0.99,1.02$ & 0.632 \\
\hline \multicolumn{4}{|l|}{ Etiology } \\
\hline Hepatitis B & 0.51 & $0.25,1.05$ & $0.067^{*}$ \\
\hline Cryptogenic & 1.06 & $0.52,2.16$ & 0.881 \\
\hline \multicolumn{4}{|l|}{ Disease characteristics at baseline } \\
\hline MELD (continuous) & 0.99 & $0.91,1.08$ & 0.827 \\
\hline Child-Pugh score & 1.50 & $0.95,2.36$ & $0.082^{*}$ \\
\hline Bilobar disease & 1.98 & $1.41,2.79$ & $<0.001^{*}$ \\
\hline Portal vein thrombosis & 1.84 & $1.21,2.80$ & $0.004^{*}$ \\
\hline Ascites & 1.56 & $1.09,2.23$ & $0.016^{\star}$ \\
\hline Total bilirubin (mg/dL) & 1.44 & $1.05,1.99$ & $0.024^{*}$ \\
\hline Creatinine (mg/dL) & 0.91 & $0.50,1.65$ & 0.758 \\
\hline \multicolumn{4}{|l|}{ Prior treatment } \\
\hline TACE & 0.68 & $0.46,1.00$ & $0.049^{*}$ \\
\hline Ablation & 0.97 & $0.55,1.70$ & 0.912 \\
\hline Resection & 0.82 & $0.45,1.50$ & 0.529 \\
\hline Radiation & 2.48 & $0.61,10.10$ & 0.206 \\
\hline Sorafenib & 2.10 & $1.37,3.22$ & $<0.001^{*}$ \\
\hline Number of SIRT procedures & 1.09 & $0.76,1.55$ & 0.645 \\
\hline Subsequent transplant & 0.08 & $0.03,0.23$ & $<0.001^{*}$ \\
\hline Dose $(\mathrm{mCi})$ administered at $1 \mathrm{st}$ SIRT & 1.48 & $1.08,2.04$ & $0.015^{*}$ \\
\hline
\end{tabular}

*, parameters are those with $\mathrm{P}<0.1$, which were entered into the multivariable model. ALBI, albumin-bilirubin; CTCAE, Common Terminology Criteria for Adverse Events; MELD, model for end-stage liver disease; NASH, nonalcoholic steatohepatitis; SIRT, selective internal radiation therapy; TACE, transarterial chemoembolization. 


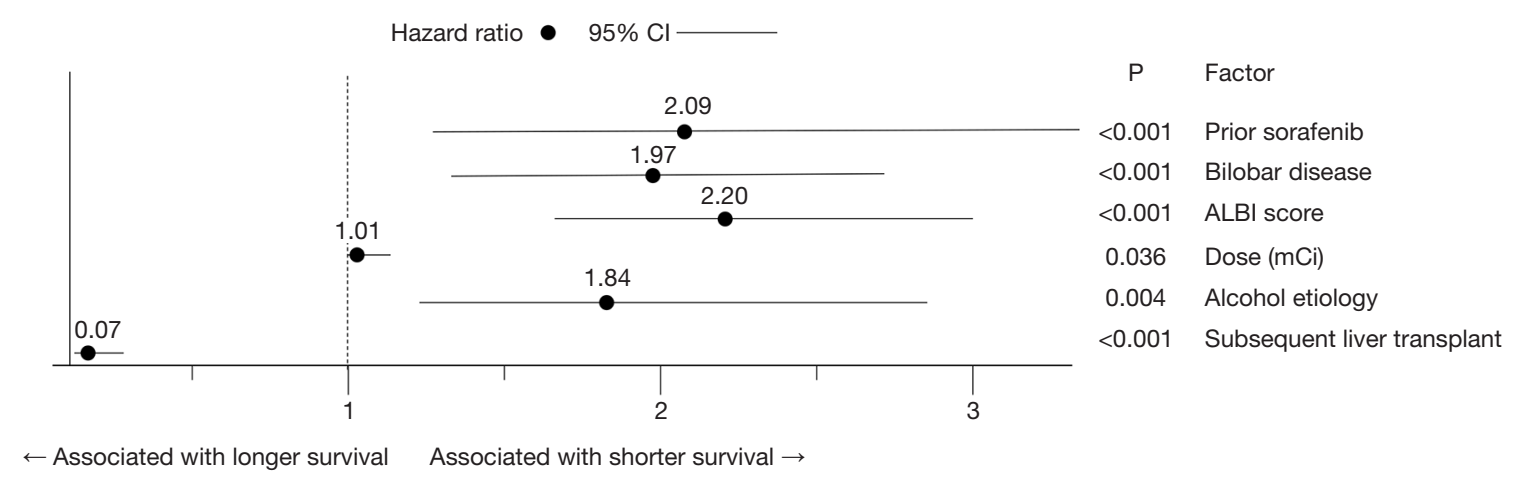

Figure 3 Factors significantly associated with OS in the multivariable Cox proportional hazards model. Hazard ratios (HRs), 95\% confidence intervals (CI), and $\mathrm{P}$ values are shown for each of the indicated factors. For the categorical variables prior sorafenib, bilobar disease, alcohol use etiology, and liver transplant, the HR represents the likelihood of death in the presence relative to the absence of these conditions. For the continuous variables ALBI score and dose, the HR represents the increase in the likelihood of death for each unit increase in the variable.

disease. At 6 months, 28 (26.7\%) had a complete response, $30(28.6 \%)$ had a partial response, 27 (25.7\%) had stable disease, and $20(19.1 \%)$ had progressive disease.

\section{Relationship of prior treatment to tumor burden and MELD score at baseline}

The majority of patients had received treatment prior to Y90 SIRT ( $\mathrm{n}=131,73.2 \%$ of the 179 with available data). The tumor burden at baseline was smaller in those who had previously undergone resection $[5.0 \mathrm{~cm}(\mathrm{n}=19) v s .6 .7 \mathrm{~cm}$ $(\mathrm{n}=150), \mathrm{P}=0.018]$ or TACE $[5.7 \mathrm{~cm}(\mathrm{n}=69)$ vs. $7.1 \mathrm{~cm}$ $(\mathrm{n}=100), \mathrm{P}=0.031]$ than in those who had not. In contrast, prior treatment with sorafenib was associated with a larger tumor burden, although the difference was not significant [7.8 cm $(\mathrm{n}=40) v s .6 .1 \mathrm{~cm}(\mathrm{n}=129) ; \mathrm{P}=0.069]$. No effect of prior ablation or radiation on tumor burden at baseline was observed.

Of the prior treatments, only resection was associated with a significant difference in MELD score at baseline [7.9 in those with prior resection $(\mathrm{n}=20) v s .9 .0$ in those without $(\mathrm{n}=154), \mathrm{P}=0.04]$.

\section{Relationship of baseline MELD score and ALBI score to administered dose}

Decreased synthetic liver function, as reflected by a higher baseline MELD score and ALBI score, correlated with lower administered dose (MELD: $\mathrm{r}=-0.17, \mathrm{P}=0.014, \mathrm{n}=215$;
ALBI: $\mathrm{r}=-0.14, \mathrm{P}=0.035, \mathrm{n}=222)$. Consistent with this, mean administered dose was higher in patients with ALBI grade $1(1.33 \pm 0.65 \mathrm{mCi})$ than in those with ALBI grades 2 $(1.10 \pm 0.37 \mathrm{mCi})$ or $3(1.14 \pm 0.28 \mathrm{mCi})$.

\section{AEs}

Of the 186 patients with data available for 1 week or 3 months after the first SIRT procedure (or both time points), 75 (40.3\%) had at least $1 \mathrm{AE}$ (Table 3). The most common AEs were abdominal pain $(n=28)$, ascites $(n=21)$, nausea $(n=12)$, and fatigue $(n=12)$. Gastrointestinal bleeding $(n=10)$, ulcers $(n=6)$, and encephalopathy $(n=5)$ were present at 3 months but not at 1 week. Severity or Common Terminology Criteria for Adverse Events grade was generally not noted in the charts and so is not included here.

Changes in laboratory values after Y90 SIRT were also recorded. Albumin decreased, and INR and total bilirubin increased significantly at 1,3 , and 6 months after the first Y90 SIRT treatment relative to baseline $(\mathrm{P}<0.001$ for all comparisons) (Table 4). Creatinine showed small increases that were significant at months 3 and 6 . The mean MELD score also increased significantly at 1,3 , and 6 months after Y90 SIRT, from $8.8 \pm 2.2$ at baseline $(\mathrm{n}=218)$ to $13.6 \pm 7.0$ at month $6(\mathrm{n}=120)(\mathrm{P}<0.001 v s$. baseline for all time points, paired $t$-test).

Although the laboratory values generally worsened after treatment, few reached severe levels. Grade 4 bilirubin values were detected in 13 patients, and grade 3 in 44 patients. No 
Table 3 Number of patients with AEs 1 week and/or 3 months after the first SIRT procedure

\begin{tabular}{lc}
\hline AE & Number with indicated AE (N=186), $\mathrm{n}(\%)$ \\
\hline Any & $75(40.3)^{\dagger}$ \\
Gastrointestinal & $28(15.1)$ \\
Abdominal pain & $21(11.3)$ \\
Ascites & $12(6.5)$ \\
Nausea & $9(4.8)$ \\
Vomiting & $8(4.3)$ \\
Decreased appetite & $6(12.4)$ \\
Ulcer & $10(5.4)$ \\
Bleeding & \\
General disorders & $9(4.8)$ \\
Edema & $12(6.5)$ \\
Fatigue & $8(4.3)$ \\
Fever & \\
Nervous system & $5(2.7)$ \\
Encephalopathy & \\
Local & \\
Groin pain & \\
Jiver & \\
Other & \\
\hline & \\
Jaundice & $(10.8)^{\ddagger}$ \\
\hline
\end{tabular}

${ }^{\dagger}$, percentages are relative to the number with data available; some patients had $>1 \mathrm{AE} .{ }^{\ddagger}$, includes abdominal distention, acute kidney injury, blisters from chemotherapy, constipation (severe), dizziness, diarrhea, duodenal stricture, gastritis, hyponatremia, myalgia, metabolic acidosis, pain/pain at site, pleural effusion, seeing colors, seizures (in a patient with prior history), shortness of breath, stomach cramps, swelling/leg swelling, weight loss. AE, adverse event; SIRT, selective internal radiation therapy.

grade 4 values were seen for other laboratory values, although grade 3 albumin $(n=5)$, grade 3 creatinine $(n=2)$, and grade 3 INR $(n=6)$ were reported.

\section{Discussion}

In this retrospective case series of 226 patients, the median OS after Y90 SIRT was 16.6 months (95\% CI, 13.1-NR), which compares favorably with that reported in recent randomized controlled trials of treatment of unresectable
HCC. The SARAH study reported a median OS of 8.0 months (95\% CI, 6.7-9.9) (23), and the SIRveNIB trial reported an OS of 8.8 months (95\% CI, 7.5-10.8) (26). Note that, in both of these prospective trials, OS was calculated from the date of randomization rather than the date of the procedure, which adds 3 to 4 weeks to the reported OS.

We examined the effects of various factors on survival using log-rank and Cox proportional hazards analyses. In the multivariable Cox model, alcohol use etiology, bilobar disease, higher ALBI score, higher administered dose, and prior sorafenib treatment predicted shorter survival after Y90 SIRT. ALBI was developed recently as a measure of liver function and, consistent with our results, has previously been associated with length of survival $(37,40)$. Notably, older age was not significantly associated with OS, suggesting that age is not a contraindication to treatment with Y90 SIRT. The associations with more-extensive disease (i.e., bilobar, higher dose), more liver dysfunction (higher ALBI score), and previous treatment with sorafenib (perhaps reflecting more widespread disease) are also consistent with our prior results from a subset of this population in which log-rank analyses associated bilobar disease, PVT, or ascites at baseline and prior sorafenib treatment with shorter survival (27). However, the much larger population in the current analysis (226 vs. 111) gives additional confidence to these results.

These results from our single-center, North American study are consistent with the results of previous multivariable analyses of Y90 SIRT in patients with unresectable HCC. In the multicenter European ENRY study, which included 325 patients, OS was 12.8 months (95\% CI, 10.9-15.7), and Eastern Cooperative Oncology Group performance status, tumor burden (number of nodules $>5$ ), INR $>1.2$, and extrahepatic disease were identified as significant predictors of shorter survival (24). Although these specific variables differed from the ones we identified, they also reflect disease severity and liver function as predictive factors, and age at treatment did not affect survival. Although the 2 study populations were similar, the ENRY study had a slightly higher proportion of patients with Child-Pugh A $(82.5 \%$ vs. $73.3 \%)$ and no patients with Child-Pugh C [compared with $3(2.0 \%)$ in the current study], lower proportions of participants who had undergone previous treatment $(41.5 \%$ vs. $73.2 \%)$ or who had HCV (44.3\% vs. $61.6 \%)$, and a higher proportion of patients with hepatitis B $(13.0 \%$ vs. $7.6 \%)$. To our knowledge, the data in the present study represent the 
Table 4 Changes in laboratory values after Y-90 SIRT

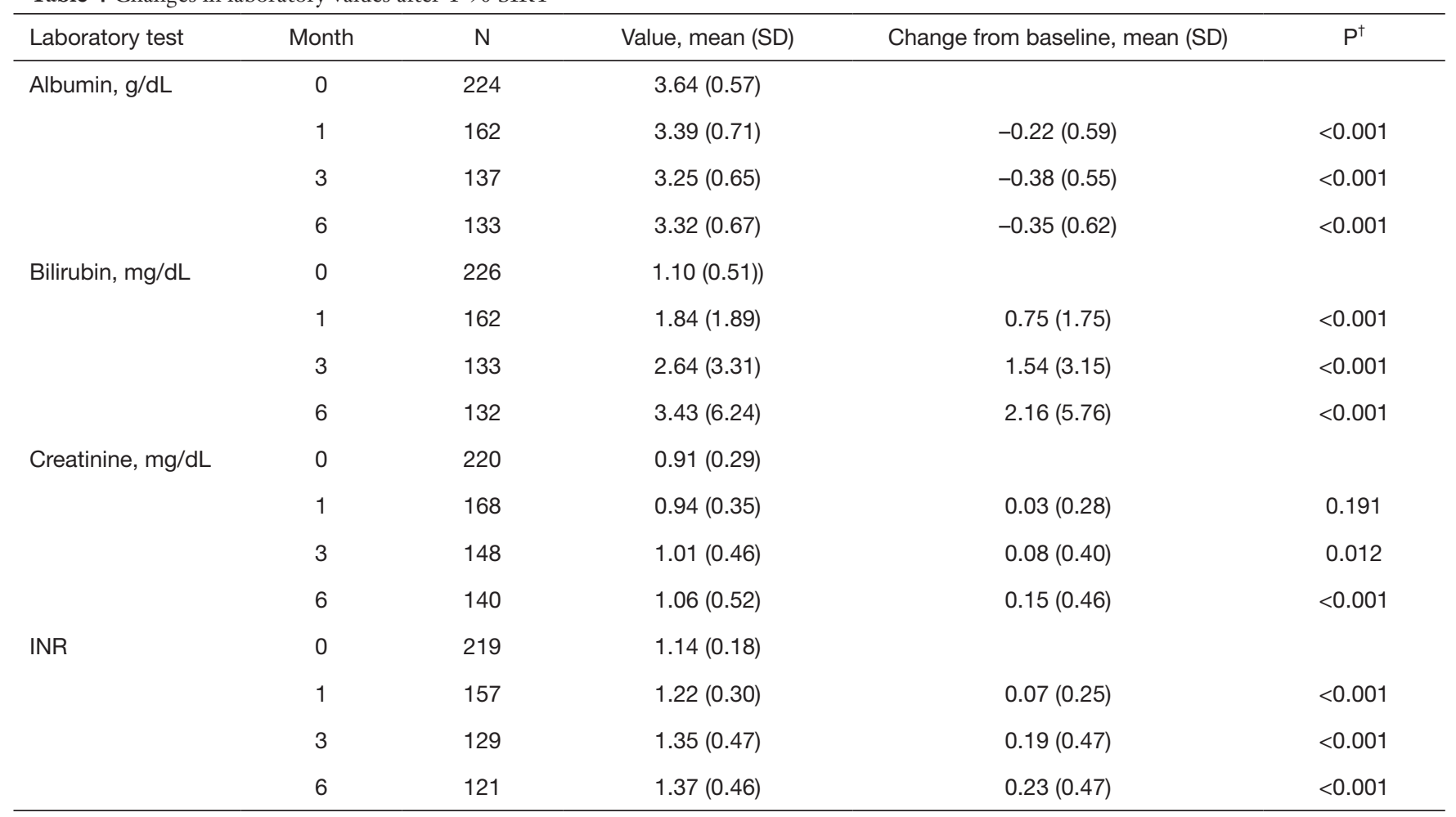

${ }^{\dagger}$, paired $t$-test. INR, international normalized ratio; SD, standard deviation; SIRT, selective internal radiation therapy; Y-90, yttrium-90.

second largest set-after the ENRY study-of prognostic factors of Y90 SIRT in HCC (24). Notably, we evaluated prognostic factors not considered by Sangro et al., such as HCC etiology, type of prior treatment, number of SIRT treatments, and dose delivered (Table S1).

In a study of Y90 SIRT in 103 patients with HCC in Singapore, Khor et al. (25) reported a median OS of 14.4 months (95\% CI, 11.0-22.2) and identified vascular invasion, $\mathrm{HCV}$ as an etiology, total bilirubin levels between 24 and $41 \mu \mathrm{mol} / \mathrm{dL}$, and serum albumin level below $28 \mathrm{~g} / \mathrm{L}$ as factors associated with shorter survival. Bilirubin and albumin as predictive factors mirror our finding of a significant association between the ALBI score and survival. However, we found that an etiology linked to alcohol use, rather than HCV, was associated with shorter OS. Although the risk of HCC remains elevated even after $\mathrm{HCV}$ is treated (41), newer treatments may preserve liver synthetic function and thereby reduce the risk of death from liver failure. In contrast, alcoholic cirrhosis is associated with a higher incidence of ongoing liver injury due to recidivism and cholestasis, and patients with alcohol use etiology are more prone to infection, all of which might lead to shorter OS $(42,43)$.

Prior sorafenib treatment continued to be associated with shorter OS in this larger cohort. In fact, only 2 of the patients in the current analysis who were treated after March 2013 (the end date for inclusion in the previous publication) had received sorafenib before their first Y90 SIRT procedure. A small study $(\mathrm{n}=10)$ examining survival in patients who had Y90 SIRT after sorafenib treatment also found lower OS (7 months) than had been reported after Y90 SIRT alone (44). A systemic treatment such as sorafenib is likely to be used in patients with more advanced disease, such as a higher tumor burden or PVT, and thus might be associated with shorter survival. Indeed, prior sorafenib treatment was associated with a numerically, but not significantly, larger tumor burden at baseline in the current study.

In contrast to prior sorafenib treatment, prior treatment with TACE or resection was associated with a significantly lower tumor burden at baseline. Prior TACE was also associated with longer survival in the Kaplan-Meier and univariate analyses, although it was not retained in the multivariable model. Consistent with our results, Golfieri 
et al. found that prior TACE significantly prolonged survival after Y90 SIRT, from 9 months to 27 months $(\mathrm{P}=0.003)$ (45). However, outcomes after Y90 SIRT in the ENRY study were similar whether or not the patient had received prior liver-directed treatment (surgery, ablation, transarterial embolization, or TACE) before Y90 SIRT, regardless of the type of prior treatment that was given $(24,46)$. We also found that any prior treatment was not significantly different from no prior treatment in our logrank analysis (17.9 vs. 13.1 months, $\mathrm{P}=0.28$; Table $\mathrm{S} 1$ ), and prior treatment did not appear to greatly affect liver function at baseline, as evaluated with the MELD score. Only prior resection correlated significantly with baseline MELD score; the score was low in patients both with and without prior resection, and the difference between the groups was not dramatic (7.9 vs. 9.0, $\mathrm{P}=0.04$ ). Together, these findings argue against a selection bias for treating patients with better prognosis after previous treatments.

By reducing tumor burden and preserving liver function, Y90 SIRT can be used as a bridge or a downstaging strategy to curative treatments (47). Twenty-six patients in our study received a transplant after the Y90 SIRT procedure, dramatically extending median survival to 69.0 months. Y90 SIRT can also be used as a bridge to resection, in part because it causes hypertrophy of the untreated lobe, making resection of the treated (lesioned) lobe more feasible $(48,49)$; however, we did not analyze subsequent resections in our patients.

Obesity and metabolic disorder can lead to NASH, which is a risk factor for HCC (11). Texas, where our center is located, has the highest rate of HCC among the 50 United States (50) and also has high rates of obesity and diabetes (51). However, NASH may be underreported in our cohort. In our experience, patients with a designated etiology of (for example) HCV may also have NASH present on biopsy without this being noted in the chart.

Many of the reported AEs were consistent with postembolization syndrome (fatigue, nausea, abdominal pain, and weight loss) $(52,53)$, but few grade 3 or 4 laboratory values were seen in the 6 months after treatment, indicating acceptable hepatotoxicity. Encephalopathy, a rare but serious complication of liver dysfunction, was seen in 5 patients (2.2\%) 3 months after Y90 SIRT. Encephalopathy has also been reported after chemotherapy and TACE (54).

The formation of ulcers due to the shunting of microspheres to the gastrointestinal tract can be a serious complication of Y90 SIRT. Ulcers were reported in only $6(2.7 \%)$ of our 226 patients, a rate similar to or lower than the rates reported in other studies, although it is possible that some cases were not captured in this retrospective study. In the ENRY study, 3.7\% of the 325 patients experienced gastrointestinal ulceration, which was responsible for 1 death (24). A systematic review of 39 studies (which mostly used resin microspheres) estimated the combined, weighted rate of ulcers to be $2.9 \%$ to $4.8 \%$ (55). A more recent systematic review and expert guidance document reported rates of $1.9 \%$ to $3.2 \%$ in a clinical trial and large retrospective cohorts (56).

This study was limited by its retrospective nature, and some data, such as the severity of most AEs, were not available. However, the large study population was diverse in terms of both demographics and disease characteristics and reflects the long experience of the authors in a realworld setting.

\section{Conclusions}

In patients with unresectable HCC treated at a single center over 13 years, use of Y90 SIRT provided an OS of 16.6 months. Alcohol use etiology, previous treatment with sorafenib, bilobar disease, and a higher ALBI score (indicating lower liver function) all predicted shorter OS, and these factors should be considered when making treatment decisions. A higher administered dose, which was associated with better liver function, was also significantly associated with shorter survival. However, more than $11 \%$ of our patients were able to receive a liver transplant after the procedure, indicating that Y90 SIRT can reduce tumor burden and maintain liver function sufficiently to allow patients to qualify for curative treatment.

\section{Acknowledgments}

The authors thank Mark Van Buskirk of Data Reduction LLC for providing statistical support and Naomi Ruff, $\mathrm{PhD}$, of Eubio LLC for providing medical writing support. Both were funded by Sirtex Medical Inc. Preliminary data from this study were presented at EASL (2019), AASLD (2018), ASTRO (2018), and ILCA (2018).

Funding: This manuscript was supported by Sirtex Medical Inc. The study received no other financial support.

\section{Footnote}

Reporting Checklist: The authors have completed the STROBE reporting checklist. Available at https://dx.doi. 
org/10.21037/jgo-20-435

Data Sharing Statement: Available at https://dx.doi. org/10.21037/jgo-20-435

Peer Review File: Available at https://dx.doi.org/10.21037/ jgo-20-435

Conflicts of Interest: All authors have completed the ICMJE uniform disclosure form (available at https://dx.doi. org/10.21037/jgo-20-435). PSM has received research funding, consulting fees, or other funds from Intercept, Abbvie, Gilead, Merck, Salix, Eisai, BMS, Sirtex, Genfit, Allergan, Celgene, Pfizer, Hepquant, Mallinckrodt, Galmed, Viking, Cymabay, Blade, Roche, LAM, and Novartis. IS has received funds from and has served as a speaker for Sirtex. The authors have no other conflicts of interest to declare.

Ethical Statement: The authors are accountable for all aspects of the work in ensuring that questions related to the accuracy or integrity of any part of the work are appropriately investigated and resolved. The study was conducted in accordance with the Declaration of Helsinki (as revised in 2013). The study was approved by the Institutional Review Board of Methodist Dallas Medical Center (No. 063.HEP.2017.D), and individual consent for this retrospective analysis was waived.

Open Access Statement: This is an Open Access article distributed in accordance with the Creative Commons Attribution-NonCommercial-NoDerivs 4.0 International License (CC BY-NC-ND 4.0), which permits the noncommercial replication and distribution of the article with the strict proviso that no changes or edits are made and the original work is properly cited (including links to both the formal publication through the relevant DOI and the license). See: https://creativecommons.org/licenses/by-nc-nd/4.0/.

\section{References}

1. American Cancer Society. Cancer Facts \& Figures 2019. Available online: https://www.cancer.org/content/ dam/cancer-org/research/cancer-facts-and-statistics/ annual-cancer-facts-and-figures/2019/cancer-facts-andfigures-2019.pdf

2. Ahmed F, Perz JF, Kwong S, et al. National trends and disparities in the incidence of hepatocellular carcinoma, 1998-2003. Prev Chronic Dis 2008;5:A74.
3. McGlynn KA, London WT. The global epidemiology of hepatocellular carcinoma: present and future. Clin Liver Dis 2011;15:223-43, vii-x.

4. Venook AP, Papandreou C, Furuse J, et al. The incidence and epidemiology of hepatocellular carcinoma: a global and regional perspective. Oncologist 2010;15 Suppl 4:5-13.

5. Siegel RL, Miller KD, Fuchs HE, et al. Cancer Statistics, 2021. CA Cancer J Clin 2021;71:7-33.

6. Gholam PM, Iyer R, Johnson MS. Multidisciplinary Management of Patients with Unresectable Hepatocellular Carcinoma: A Critical Appraisal of Current Evidence. Cancers (Basel) 2019;11:873.

7. Wang CY, Li S. Clinical characteristics and prognosis of 2887 patients with hepatocellular carcinoma: A single center 14 years experience from China. Medicine (Baltimore) 2019;98:e14070.

8. Toyoda H, Kumada T, Tada T, et al. Differences in the impact of prognostic factors for hepatocellular carcinoma over time. Cancer Sci 2017;108:2438-44.

9. Tandon P, Garcia-Tsao G. Prognostic indicators in hepatocellular carcinoma: a systematic review of 72 studies. Liver Int 2009;29:502-10.

10. Adhoute X, Penaranda G, Raoul JL, et al. Usefulness of staging systems and prognostic scores for hepatocellular carcinoma treatments. World J Hepatol 2016;8:703-15.

11. Lafaro KJ, Demirjian AN, Pawlik TM. Epidemiology of hepatocellular carcinoma. Surg Oncol Clin N Am 2015;24:1-17.

12. El-Serag HB, Kanwal F. Epidemiology of hepatocellular carcinoma in the United States: where are we? Where do we go? Hepatology 2014;60:1767-75.

13. European Association for the Study of the Liver. Electronic address: easloffice@easloffice.eu; European Association for the Study of the Liver. EASL Clinical Practice Guidelines: Management of hepatocellular carcinoma. J Hepatol 2018;69:182-236.

14. National Comprehensive Cancer Network. NCCN clinical practice guidelines in oncology: Hepatobiliary cancers version 1.2019. 2019. Available online: https://www.nccn. org/professionals/physician_gls/pdf/hepatobiliary.pdf

15. Braat AJ, Huijbregts JE, Molenaar IQ, et al. Hepatic radioembolization as a bridge to liver surgery. Front Oncol 2014;4:199.

16. Lang L. FDA approves sorafenib for patients with inoperable liver cancer. Gastroenterology 2008;134:379.

17. US Food and Drug Administration. FDA approves lenvatinib for unresectable hepatocellular carcinoma. August 16, 2018. Available online: https://www.fda.gov/drugs/ 
informationondrugs/approveddrugs/ucm617185.htm

18. US Food and Drug Administration. FDA grants accelerated approval to nivolumab for HCC previously treated with sorafenib. Available online: https://www.fda. gov/drugs/resources-information-approved-drugs/fdagrants-accelerated-approval-nivolumab-hcc-previouslytreated-sorafenib

19. US Food and Drug Administration. FDA grants accelerated approval to pembrolizumab for hepatocellular carcinoma. Available online: https://www.fda.gov/ drugs/fda-grants-accelerated-approval-pembrolizumabhepatocellular-carcinoma

20. Yau T, Kang YK, Kim TY, et al. Efficacy and Safety of Nivolumab Plus Ipilimumab in Patients With Advanced Hepatocellular Carcinoma Previously Treated With Sorafenib: The CheckMate 040 Randomized Clinical Trial. JAMA Oncol 2020;6:e204564.

21. Likhitsup A, Razumilava N, Parikh ND. Treatment for Advanced Hepatocellular Carcinoma: Current Standard and the Future. Clin Liver Dis (Hoboken) 2019;13:13-9.

22. Bouvry C, Palard X, Edeline J, et al. Transarterial Radioembolization (TARE) Agents beyond 90Y-Microspheres. Biomed Res Int 2018;2018:1435302.

23. Vilgrain V, Pereira H, Assenat E, et al. Efficacy and safety of selective internal radiotherapy with yttrium-90 resin microspheres compared with sorafenib in locally advanced and inoperable hepatocellular carcinoma (SARAH): an open-label randomised controlled phase 3 trial. Lancet Oncol 2017;18:1624-36.

24. Sangro B, Carpanese L, Cianni R, et al. Survival after yttrium-90 resin microsphere radioembolization of hepatocellular carcinoma across Barcelona clinic liver cancer stages: a European evaluation. Hepatology 2011;54:868-78.

25. Khor AY, Toh Y, Allen JC, et al. Survival and pattern of tumor progression with yttrium-90 microsphere radioembolization in predominantly hepatitis B Asian patients with hepatocellular carcinoma. Hepatol Int 2014;8:395-404.

26. Chow PKH, Gandhi M, Tan SB, et al. SIRveNIB: Selective Internal Radiation Therapy Versus Sorafenib in Asia-Pacific Patients With Hepatocellular Carcinoma. J Clin Oncol 2018;36:1913-21.

27. Mantry PS, Mehta A, Madani B, et al. Selective internal radiation therapy using yttrium-90 resin microspheres in patients with unresectable hepatocellular carcinoma: a retrospective study. J Gastrointest Oncol 2017;8:799-807.

28. Palmer DH, Hawkins NS, Vilgrain V, et al. Tumor burden and liver function in HCC patient selection for selective internal radiation therapy: SARAH post-hoc study. Future Oncol 2020;16:4315-25.

29. Oladeru OT, Miccio JA, Yang J, et al. Conformal external beam radiation or selective internal radiation therapy-a comparison of treatment outcomes for hepatocellular carcinoma. J Gastrointest Oncol 2016;7:433-40.

30. Paprottka KJ, Schoeppe F, Ingrisch M, et al. Pretherapeutic factors for predicting survival after radioembolization: a single-center experience in 389 patients. Eur J Nucl Med Mol Imaging 2017;44:1185-93.

31. Jeliazkova P, Umgelter A, Braren R, et al. Prognostic factors in hepatocellular carcinoma patients undergoing transarterial chemoembolization and radioembolization: a retrospective study. Eur J Gastroenterol Hepatol 2020;32:1036-41.

32. Iñarrairaegui $M$, Martinez-Cuesta $A$, Rodríguez $M$, et al. Analysis of prognostic factors after yttrium-90 radioembolization of advanced hepatocellular carcinoma. Int J Radiat Oncol Biol Phys 2010;77:1441-8.

33. Floridi C, Pesapane F, Angileri SA, et al. Yttrium-90 radioembolization treatment for unresectable hepatocellular carcinoma: a single-centre prognostic factors analysis. Med Oncol 2017;34:174.

34. Spreafico C, Sposito C, Vaiani M, et al. Development of a prognostic score to predict response to Yttrium-90 radioembolization for hepatocellular carcinoma with portal vein invasion. J Hepatol 2018;68:724-32.

35. Weng Z, Ertle J, Zheng S, et al. A new model to estimate prognosis in patients with hepatocellular carcinoma after Yttrium-90 radioembolization. PLoS One 2013;8:e82225.

36. SIR-Spheres Y-90 resin microspheres (yttrium-90 microspheres). North Sydney, New South Wales, Australia: Sirtex Medical Limited, 2017.

37. Johnson PJ, Berhane S, Kagebayashi C, et al. Assessment of liver function in patients with hepatocellular carcinoma: a new evidence-based approach-the ALBI grade. J Clin Oncol 2015;33:550-8.

38. Therasse P, Arbuck SG, Eisenhauer EA, et al. New guidelines to evaluate the response to treatment in solid tumors. European Organization for Research and Treatment of Cancer, National Cancer Institute of the United States, National Cancer Institute of Canada. J Natl Cancer Inst 2000;92:205-16.

39. Lencioni R, Llovet JM. Modified RECIST (mRECIST) assessment for hepatocellular carcinoma. Semin Liver Dis 2010;30:52-60.

40. Mohammadi $\mathrm{H}$, Abuodeh $\mathrm{Y}$, Jin $\mathrm{W}$, et al. Using the 
Albumin-Bilirubin (ALBI) grade as a prognostic marker for radioembolization of hepatocellular carcinoma. J Gastrointest Oncol 2018;9:840-6.

41. El-Serag HB, Kanwal F, Richardson P, et al. Risk of hepatocellular carcinoma after sustained virological response in Veterans with hepatitis $\mathrm{C}$ virus infection. Hepatology 2016;64:130-7.

42. Jüngst C, Berg T, Cheng J, et al. Intrahepatic cholestasis in common chronic liver diseases. Eur J Clin Invest 2013;43:1069-83.

43. Arab JP, Roblero JP, Altamirano J, et al. Alcohol-related liver disease: Clinical practice guidelines by the Latin American Association for the Study of the Liver (ALEH). Ann Hepatol 2019;18:518-35.

44. Rana N, Ju AW, Bazylewicz M, et al. Yttrium-90 Radioembolization in Patients with Hepatocellular Carcinoma Who have Previously Received Sorafenib. Front Oncol 2013;3:323.

45. Golfieri R, Bilbao JI, Carpanese L, et al. Comparison of the survival and tolerability of radioembolization in elderly vs. younger patients with unresectable hepatocellular carcinoma. J Hepatol 2013;59:753-61.

46. Sangro B, Maini CL, Ettorre GM, et al. Radioembolisation in patients with hepatocellular carcinoma that have previously received liver-directed therapies. Eur J Nucl Med Mol Imaging 2018;45:1721-30.

47. Lewandowski RJ, Kulik LM, Riaz A, et al. A comparative analysis of transarterial downstaging for hepatocellular carcinoma: chemoembolization versus radioembolization. Am J Transplant 2009;9:1920-8.

48. Gabr A, Abouchaleh N, Ali R, et al. Outcomes of Surgical Resection after Radioembolization for Hepatocellular

Cite this article as: Shah RM, Sheikh S, Shah J, Vivian E, Mejia A, Shahin I, Mantry PS. Prognostic factors of unresectable hepatocellular carcinoma treated with yttrium-90 radioembolization: results from a large cohort over 13 years at a single center. J Gastrointest Oncol 2021;12(4):1718-1731. doi: 10.21037/jgo-20-435
Carcinoma. J Vasc Interv Radiol 2018;29:1502-10.e1.

49. Garlipp B, de Baere T, Damm R, et al. Left-liver hypertrophy after therapeutic right-liver radioembolization is substantial but less than after portal vein embolization. Hepatology 2014;59:1864-73.

50. White DL, Thrift AP, Kanwal F, et al. Incidence of Hepatocellular Carcinoma in All 50 United States, From 2000 Through 2012. Gastroenterology 2017;152:81220.e5.

51. Robert Wood Johnson Foundation. The state of obesity in Texas. Available online: https://www.stateofobesity.org/ states/tx

52. Orwat KP, Beckham TH, Cooper SL, et al. Pretreatment albumin may aid in patient selection for intrahepatic Y-90 microsphere transarterial radioembolization (TARE) for malignancies of the liver. J Gastrointest Oncol 2017;8:1072-8.

53. Padia SA, Lewandowski RJ, Johnson GE, et al. Radioembolization of Hepatic Malignancies: Background, Quality Improvement Guidelines, and Future Directions. J Vasc Interv Radiol 2017;28:1-15.

54. Willson KJ, Nott LM, Broadbridge VT, et al. Hepatic encephalopathy associated with cancer or anticancer therapy. Gastrointest Cancer Res 2013;6:11-6.

55. Naymagon S, Warner RR, Patel K, et al. Gastroduodenal ulceration associated with radioembolization for the treatment of hepatic tumors: an institutional experience and review of the literature. Dig Dis Sci 2010;55:2450-8.

56. Sangro B, Martínez-Urbistondo D, Bester L, et al. Prevention and treatment of complications of selective internal radiation therapy: Expert guidance and systematic review. Hepatology 2017;66:969-82. 
Table S1 Kaplan-Meier survival analyses

\begin{tabular}{|c|c|c|c|c|c|}
\hline Variable & Category & $\mathrm{n}$ & OS (months) & $95 \% \mathrm{Cl}$ & P (log-rank) \\
\hline All patients & All patients & 226 & 16.6 & 13.1, NR & \\
\hline \multicolumn{6}{|l|}{ Demographics } \\
\hline \multirow[t]{2}{*}{ Sex } & Female & 52 & 16.6 & $8.3,20.8$ & 0.252 \\
\hline & Male & 174 & 17.9 & $13.1,24.4$ & \\
\hline \multirow[t]{2}{*}{ White } & Caucasian & 133 & 17.2 & $13.1,23.5$ & 0.559 \\
\hline & Non-Caucasian & 93 & 15.2 & $11.0,25.3$ & \\
\hline \multirow[t]{2}{*}{ Age 65 (years) } & $<65$ & 112 & 16.2 & $11.9,21.0$ & 0.675 \\
\hline & $\geq 65$ & 114 & 18.4 & $12.8,25.1$ & \\
\hline \multirow[t]{2}{*}{ Age 70 (years) } & $<70$ & 156 & 16.3 & $12.2,19.7$ & 0.796 \\
\hline & $\geq 70$ & 70 & 21.0 & $12.4,27.8$ & \\
\hline \multicolumn{6}{|l|}{ Etiology } \\
\hline \multirow[t]{2}{*}{ Hepatitis B } & Yes & 17 & 47.4 & $11.0, \mathrm{NR}$ & 0.062 \\
\hline & No & 207 & 16.3 & $12.5,20.8$ & \\
\hline \multirow[t]{2}{*}{ Hepatitis C } & Yes & 138 & 16.6 & $11.9,25.0$ & 0.884 \\
\hline & No & 86 & 16.6 & $13.1,24.4$ & \\
\hline \multirow[t]{2}{*}{ Alcohol use $^{\dagger}$} & Yes & 45 & 11.8 & $7.9,23.5$ & $0.021^{\circ}$ \\
\hline & No & 179 & 18.6 & $15.6,24.4$ & \\
\hline \multirow[t]{2}{*}{$\mathrm{NASH}$} & Yes & 27 & 16.6 & $15.2,27.8$ & 0.663 \\
\hline & No & 197 & 16.7 & $12.3,23.6$ & \\
\hline \multicolumn{6}{|c|}{ Disease characteristics at baseline } \\
\hline \multirow[t]{2}{*}{ MELD } & $<8$ & 91 & 16.6 & $12.1,23.5$ & 0.301 \\
\hline & $\geq 8$ & 127 & 19.7 & $11.8,27.8$ & \\
\hline \multirow[t]{2}{*}{ ALBI score ${ }^{\dagger}$ (median) } & $<-2.28$ & 113 & 23.5 & $16.3,26.1$ & 0.043 \\
\hline & $\geq-2.28$ & 113 & 12.4 & $9.4,17.2$ & \\
\hline \multirow[t]{3}{*}{ ALBI grade } & 1 & 74 & 23.8 & $16.6,42.5$ & 0.091 \\
\hline & 2 & 136 & 14.8 & $11.4,19.3$ & \\
\hline & 3 & 16 & 10.4 & $5.6,25.1$ & \\
\hline \multirow[t]{2}{*}{ Child-Pugh score } & A & 110 & 17.9 & $13.1,27.8$ & 0.081 \\
\hline & $B / C$ & 40 & 9.6 & $6.4,13.5$ & \\
\hline \multirow[t]{2}{*}{ Bilobar disease $^{\dagger}$} & Yes & 105 & 10.8 & $8.5,15.2$ & $<0.001^{*}$ \\
\hline & No & 116 & 25.0 & $16.7,29.7$ & \\
\hline Portal vein thrombosis & Yes & 44 & 11.0 & $8.0,13.9$ & $0.004^{*}$ \\
\hline & No & 160 & 19.3 & $15.6,26.1$ & \\
\hline Ascites & Yes & 71 & 12.0 & $8.4,17.9$ & $0.015^{\circ}$ \\
\hline & No & 140 & 20.8 & $16.2,26.1$ & \\
\hline Laboratory values at baseline & C grade) & & & & \\
\hline Albumin CTC & 0 & 144 & 19.7 & $16.2,26.1$ & 0.069 \\
\hline & 1 & 52 & 12.2 & $8.6,17.2$ & \\
\hline & 2 & 30 & 9.4 & $6.9,25.1$ & \\
\hline Total bilirubin CTC & 0 & 114 & 18.6 & $16.2,25.7$ & 0.287 \\
\hline & 1 & 65 & 13.5 & $8.7,26.1$ & \\
\hline & 2 & 46 & 11.8 & $8.1,20.8$ & \\
\hline & 3 & 1 & 8.0 & - & \\
\hline Prior treatment & & & & & \\
\hline Any & Yes & 131 & 17.9 & $12.8,27.8$ & 0.278 \\
\hline & No & 48 & 13.1 & $7.1,23.8$ & \\
\hline TACE & Yes & 74 & 25.3 & $13.9,29.8$ & $0.047^{*}$ \\
\hline & No & 105 & 13.5 & $10.3,20.8$ & \\
\hline Ablation & Yes & 25 & 27.8 & $10.3,35.3$ & 0.909 \\
\hline & No & 154 & 16.7 & $12.3,23.8$ & \\
\hline Resection & Yes & 21 & 16.3 & $12.0,42.5$ & 0.528 \\
\hline & No & 158 & 16.7 & $12.1,25.1$ & \\
\hline Sorafenib ${ }^{\dagger}$ & Yes & 40 & 10.3 & $5.3,12.5$ & $<0.001^{*}$ \\
\hline & No & 139 & 23.8 & $16.2,27.8$ & \\
\hline Number of SIRT treatments & 1 & 157 & 16.7 & $12.4,25.0$ & 0.644 \\
\hline & $2+$ & 69 & 16.6 & $11.0,23.6$ & \\
\hline Radiation dose $\mathrm{e}^{\dagger}$ (category) & $<1.1 \mathrm{mCi}$ & 108 & 16.6 & $12.5,29.8$ & 0.079 \\
\hline & $\geq 1.1 \mathrm{mCi}$ & 114 & 16.7 & $11.8,20.8$ & \\
\hline Subsequent transplant ${ }^{\dagger}$ & Yes & 26 & 69.0 & $43.9,69.0$ & $<0.001^{\circ}$ \\
\hline & No & 200 & 13.9 & $11.8,16.7$ & \\
\hline
\end{tabular}

${ }^{\dagger}$, included in the final multivariable model. ${ }^{*}, \mathrm{P}<0.05$. ALBI, albumin-bilirubin; $\mathrm{Cl}$, confidence interval; CTC, Common Terminology Criteria; MELD, model for end-stage liver disease; NASH, nonalcoholic steatohepatitis; NR, not reached; OS, overall survival; SIRT, selective internal radiation therapy; TACE, transarterial chemoembolization. 
Table S2 OS multivariable Cox proportional hazards model with transplant as time-dependent covariate: sensitivity analysis on impact of missing data by multiple imputation

\begin{tabular}{|c|c|c|c|c|c|c|}
\hline Parameter & \multicolumn{3}{|c|}{ Original results } & \multicolumn{3}{|c|}{ Multiple imputation results } \\
\hline Sorafenib & 2.09 & $1.35-3.23$ & $<0.001^{*}$ & 2.05 & $1.32-3.17$ & $0.001^{*}$ \\
\hline Transplant & 0.07 & $0.02-0.20$ & $<0.001^{*}$ & 0.05 & $0.02-0.15$ & $<0.001^{*}$ \\
\hline Bilobar disease & 1.97 & $1.38-2.82$ & $<0.001^{*}$ & 1.91 & $1.33-2.75$ & $<0.001^{*}$ \\
\hline Dose (mCi) & 1.01 & $1.00-1.03$ & $0.036^{*}$ & 1.01 & $1.00-1.02$ & 0.105 \\
\hline ALBI score & 2.20 & $1.62-2.98$ & $<0.001^{*}$ & 2.17 & $1.60-2.94$ & $<0.001^{\star}$ \\
\hline
\end{tabular}

*, $\mathrm{P}<0.05$. ALBI, albumin-bilirubin; $\mathrm{Cl}$, confidence interval; HR, hazard ratio. 